OPEN ACCESS

Edited by: Uday Kishore,

Brunel University London, United Kingdom

Reviewed by:

Jorg Hermann Fritz,

McGill University, Canada

Eswari Dodagatta-Marri,

University of California, San Francisco,

United States

${ }^{*}$ Correspondence:

Kathleen E. Rodgers

krodgers@email.arizona.edu

Specialty section

This article was submitted to

Molecular Innate Immunity,

a section of the journal

Frontiers in Immunology

Received: 10 September 2019

Accepted: 25 November 2019

Published: 10 December 2019

Citation:

Soto M, Gaffney KJ and Rodgers KE (2019) Improving the Innate Immune

Response in Diabetes by Modifying the Renin Angiotensin System.

Front. Immunol. 10:2885 doi: 10.3389/fimmu.2019.02885

\section{Improving the Innate Immune Response in Diabetes by Modifying the Renin Angiotensin System}

\author{
Maira Soto, Kevin J. Gaffney and Kathleen E. Rodgers* \\ Pharmacology Department, College of Medicine, Center for Innovation in Brain Science, University of Arizona, Tucson, AZ, \\ United States
}

Patients with Type 2 Diabetes Mellitus (T2DM) suffer from a higher incidence and severity of pulmonary infections. This is likely due to immune impairment and structural abnormalities caused by T2DM-induced oxidative stress (OS) and chronic inflammation. Modulation of the Renin Angiotensin System (RAS) through blockade of the actions of angiotensin II (All), or inducing the protective pathway, has the potential to reduce these pathological pathways. The effects of Angiotensin 1-7 [A(1-7)] and NorLeu ${ }^{3}-A(1-7)$ [NorLeu], ligands of the protective RAS, on the innate immune response were evaluated in the $d b / d b$ mouse model of T2DM. Only NorLeu treatment reduced the structural pathologies in the lung caused by T2DM. A decreased in bactericidal activity and phagocytosis in diabetic animals was also observed; both $A(1-7)$ and NorLeu treatment restored these functions. Myeloid progenitor CFUs were reduced and neutrophil/progenitor OS was increased in saline-treated $d b / d b$ mice, and was reversed by $A(1-7)$ and NorLeu treatment. These results demonstrate the adverse effects of diabetes on factors that contribute to pulmonary infections and the therapeutic potential of protective RAS peptides. Overall, RAS-modification may be a viable therapeutic target to treat diabetic complications that are not addressed by glucose lowering drugs.

Keywords: diabetes, immunosuppression, innate, PMNs, alveolar-macrophages, angiotensin, NorLeu

\section{INTRODUCTION}

T2DM is a worldwide epidemic. In the United States alone, 23.2 million people (7.5\% of the population) are currently diagnosed with T2DM. T2DM is now the seventh leading cause of death (1). The collective impact of T2MD-associated complications results in both reduced quality of life and increased economic burden (2).

In addition to the well-recognized complications and co-morbidities associated with T2DM, diabetic patients also suffer from less-studied complications such as decreased lung function and impaired immune function (3-7). Diabetic patients are more likely to suffer from infection and at a greater risk of complications after infection (8-12). During the 2009 H1N1 Influenza pandemic, diabetic patients had a higher rate of hospitalizations (13-15). Diabetes is also linked to higher rates of Methicillin-resistant Staphylococcus aureus (MRSA) infection (7). Epidemiological data links diabetes to higher incidence of a variety of cancers, including liver, pancreas and lymphoma; perhaps due to immune suppression (1). Mouse models of hind paw infection show diminished innate immunity at the site of infection and reduced circulating polymorphonuclear leukocytes (PMN) function in diabetic mice (3). PMN counts can be affected by metabolic parameters such as age, BMI, and systolic blood pressure (16). Increases in the frequency of all these ailments 
in diabetic patients and diabetic mouse models indicate there is a decrease in the activity of the cells that are involved in innate immunity. Dysregulation of PMNs is associated with several diabetic complications, such as hypofibrinolysis, nephropathy and cardiovascular events (17-19). Immune-suppression in diabetic patients happens despite the availability of current glucose control medications for T2DM, highlighting a need for additional therapeutic intervention.

Traditionally, the Renin Angiotensin System (RAS) is known for its role in blood pressure regulation. Both angiotensin II (AII) and angiotensin (1-7) [A(1-7)] are bioactive peptides of RAS; both of these peptides have now been associated with physiological functions that reach beyond the regulation of hypertension. Activation of the angiotensin type I (AT1) receptor by AII results in a number of pathological processes including vasoconstriction, increased pro-inflammatory response, elevated levels of oxidative stress (OS), insulin resistance, hypertension (HTN), and end organ failure (9-12, 20). A(1-7), acting primarily through Mas receptor activation, causes vasodilation, decreased OS and has anti-inflammatory effects $(4,6,13)$. These actions of the protective RAS may reduce co-morbidities related to T2DM. The discovery of these protective effects by RASmodifying peptides has prompted therapeutic interest in this system. NorLeu ${ }^{3}-\mathrm{A}(1-7)$ [NorLeu], a peptide analog of A(1-7), has already shown efficacy in diabetic wound repair (21-25).

Studies reported herein were designed to further understand the impact of T2DM and RAS modification on immune parameters that are important in clearing respiratory infections, using Staphylococcus aureus (S. aureus) as a pathogenic model in $d b / d b$ mice. The primary mode of $S$. aureus clearance in this pneumonia model is through alveolar macrophages and neutrophils $(26,27)$, both main players in pulmonary innate immunity. As chronic inflammation and OS may contribute to the potential immunosuppression in diabetics, $A(1-7)$ and NorLeu were used as novel treatments to correct diabetesinduced immune dysfunction in the $d b / d b$ model.

\section{MATERIALS AND METHODS}

\section{Animal Procedures}

Male BKS.Cg-Dock7 $7^{\mathrm{m}}+/+\operatorname{Lepr}^{\mathrm{db}} / \mathrm{J}(d b / d b)$ mice and agematched non-diabetic heterozygous controls ( $h t z)$ were purchased from Jackson Laboratories (Bar Harbor, ME, USA). Eight week old mice were randomized into three treatment groups ( $n=6-10$ /group). Animals were administered either saline ( $h t z$ and $d b / d b), \mathrm{A}(1-7)(0.5 \mathrm{mg} / \mathrm{kg} / \mathrm{day})(d b / d b)$, or NorLeu $(0.5 \mathrm{mg} / \mathrm{kg} /$ day) ( $d b / d b)$ subcutaneously, daily for 4,8 , or 12 weeks. Dose finding studies previously performed indicated $0.5 \mathrm{mg} / \mathrm{kg} /$ day is optimal (28). Pharmaceutical grade A(1-7) and NorLeu were purchased from Bachem (Torrance, CA, USA). Mice were kept on a $12 \mathrm{~h}$ light/dark cycle and food and water were available ad libitum. Body weight was assessed at necropsy. Blood glucose level was measured using a Free Style Lite meter (Abbott Laboratories, Abbott Park, IL) from a drop of blood obtained from the saphenous vein. All animal studies have been reviewed and approved by the University of Southern California Institutional Animal Care and Use Committee (IACUC).

\section{Micro-CT Scanning and Analysis}

The Inveon preclinical CT scanner (Siemens, Knoxville, TN) was used for data-acquisition in prone position under $2 \%$ isoflurane inhalation anesthesia (tube voltage $80 \mathrm{kV}$, tube current $500 \mu \mathrm{A}, 0.104 \mathrm{~mm}$ effective pixel size, binning of 2 to reduce noise) with and without respiratory gating (i.e., synchronization of acquisition of micro-CT projections with a time-point in the respiratory cycle of the individual mouse). Scanning took 10 and $30 \mathrm{~min}$ with and without respiratory gating, respectively. Respiratory monitoring was performed using a pressure transducer pad (System BioVet ${ }^{\mathrm{TM}} \Subset \mathrm{m} 2 \mathrm{~m}$ Imaging Corp, Newark, USA) placed under the animal's chest. Images were reconstructed and assessed at a constant window width/window level (5000/2000). The acquired images were reconstructed using the Inveon ${ }^{\mathrm{TM}}$ Acquisition Workplace software (Siemens, Knoxville, TN). A Feldkamp algorithm with a Shepp and Logan filter was used to reconstruct the acquired images. The images were output and stored in a dicom format. The CT image data were analyzed using AMIRA (FEI, Visualization Sciences Group, Houston, TX) to create volume renderings. 3 -D segmentation of lung tissue and airway was performed based on gray value threshold difference between tissue and air. Volumes of different lung segments at both inhalation and expiration were automatically quantified by AMIRA. A ratio of inhalation and expiration volumes was used as indirect marker for lung compliance.

\section{S. aureus Survival Assay}

$S$. aureus strain Newman was provided by Dr. Annie WongBeringer's laboratory (USC). After 6-weeks of treatment, blood was collected from the tail-vein and placed in microvette heparin coated tubes. A 1:10 bacterial solution was prepared, incubated with agitation for $30 \mathrm{~min}$ at $37^{\circ} \mathrm{C}$ and then diluted into fresh $\mathrm{DMEM}+5 \%$ fetal bovine serum (FBS) at 1:12. For each animal, $25 \mu \mathrm{L}$ of blood diluted into $162.5 \mu \mathrm{L}$ S. aureus $\left(6 \times 10^{6} \mathrm{CFUs}\right)$ preparation and incubated at $37^{\circ} \mathrm{C}$ in a rotating platform for 30 min. Surviving $S$. aureus titers were determined by plating serial dilutions in duplicate on tryptic soy agar (TSA) plates with $5 \%$ sheep blood.

\section{Neutrophil Activity Assays}

Twenty microliters of blood were collected into a heparinized tube from the mouse tail vein, the RBCs were lysed and remaining cells were washed and suspended in DMEM+5\%FBS. Samples were placed on a 96-well flat bottom plate and $100 \mu \mathrm{L}$ of $1 \mathrm{mg} / \mathrm{mL}$ pHrodo Red S. aureus BioParticles Conjugate (pHrodo) in DMEM $+5 \%$ FBS and $0.5 \mu \mathrm{L}$ of CellROX OS Reagents (Thermo Fisher Scientific) were added. The $S$. aureus were added in excess to capture maximal phagocytic capabilities. The plate was read at $10 \mathrm{~min}$ intervals for $110 \mathrm{~min}$ at $\mathrm{Ex}$ 509/Em 533 (pHrodo) and Ex 640/Em 665 (CellROX) on a Synergy H1 Hybrid Multi-Mode Microplate Reader (BioTek, Winooski, VT). These samples were also read on a LSR II flow cytometer (BD Biosciences, San Jose, CA). Data were analyzed using FlowJo V 10.0.7r2. 


\section{Neutrophil Phagocytosis Assay}

Heparinized blood was collected from the tail vein of treated mice, and total WBCs were counted. Twenty microliters of whole blood were diluted 1:5 with DMEM+5\%FBS, $5 \mu \mathrm{L}$ of $1 \mathrm{mg} / \mathrm{mL}$ pHrodo was added and incubated at $37^{\circ} \mathrm{C}$ for $90 \mathrm{~min}$. RBCs were lysed, the sample was washed and then fixed with $10 \%$ buffered formalin. Samples were read on a LSR II flow cytometer. Data were analyzed using FlowJo V 10.0.7r2 to determine the total concentration of PMNs and phagocytic PMNs in whole blood.

\section{Plasma Collection}

At the necropsy, mice were overdosed with ketamine/xylazine and the blood was collected by cardiac puncture into EDTAcoated tubes. Immediately after collection plasma was isolated by centrifugation; plasma aliquots were stored at $-80^{\circ} \mathrm{C}$ for later analysis.

\section{Plasma Cytokine Measurements}

Plasma collected at necropsy was also used to measure circulating cytokine levels using the V-PLEX Proinflammatory Panel 1 Mouse Kit (Meso Scale Diagnostics; Rockville, MD).

\section{Bone Marrow (BM) Collection and Cultures}

Femurs from un-infected mice were collected after 4, 8, and 12 weeks of treatment and BM was harvested by flushing with PBS containing 2\%FBS/2x Pen/Strep. BM cells were washed, counted and re-suspended in DMEM+5\%FBS at $5 \times 10^{6}$ cells $/ \mathrm{mL}$ Mouse MethoCult ${ }^{\mathrm{TM}}$ media (StemCell Technologies, Cambridge, MA), $900 \mu \mathrm{L}$, was added 24-well tissue culture plates, $20 \mu \mathrm{L}$ of cells mixed in and cultures incubated at $37^{\circ} \mathrm{C}, 5 \% \mathrm{CO}_{2}$ in air. After 12 days of culture, CFU-granulocyte, erythrocyte, macrophage and megakaryocyte (GEMM) and CFU- granulocyte macrophage (GM) colonies were counted under phase contrast microscopy.

\section{3-Nitrotyrosine (N-Tyr) Staining}

BM cells were fixed with $4 \%$ paraformaldehyde, incubated with permeabilizing solution ( $0.1 \%$ Triton-X in PBS) for $15 \mathrm{~min}$ in the dark at room temperature, and washed. BM cells in 100 $\mu \mathrm{L}$ of $2 \%$ FBS in PBS were stained with $1 \mu \mathrm{L}$ L anti-NTyrosine-FITC antibody (EMD Millipore Corp, Temecula, CA; Clone 1A6) $30 \mathrm{~min}$ at $\mathrm{RT}$, washed, resuspended in $0.5 \mathrm{~mL}$ of $1 \%$ paraformaldehyde and stored in the dark at $4{ }^{\circ} \mathrm{C}$ until flow cytometric analysis. Percentage of PMNs, N-Tyr+ cells and NTyr+ PMNs were calculated from this analysis.

\section{Histological Analysis}

At the necropsy lungs were rapidly excised and weighed. The right lobe was formalin-fixed and paraffin-embedded, cut at $7 \mu \mathrm{m}$ and stained with Hematoxylin and Eosin. Four to five images per

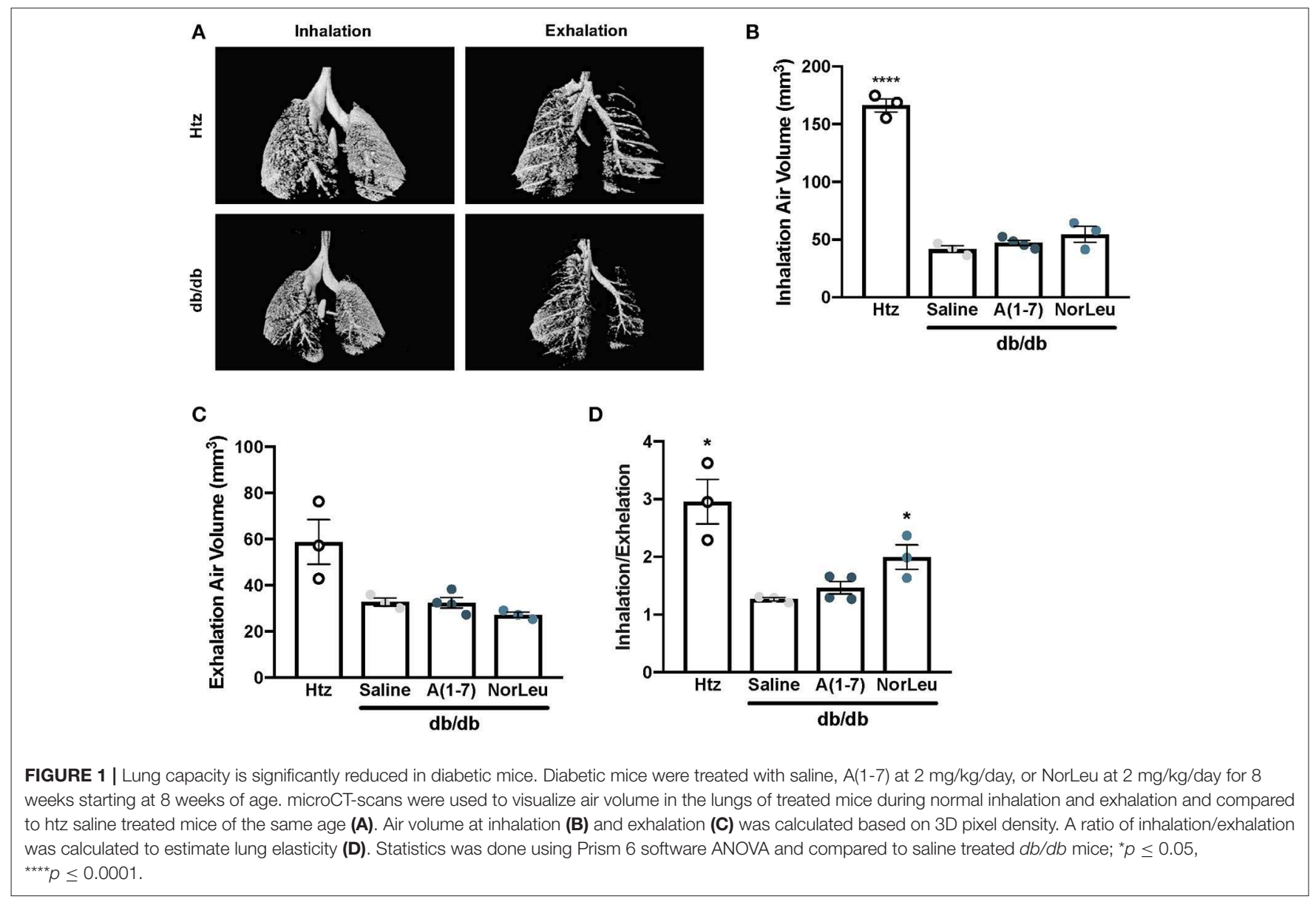


slide were taken at 40x magnification, 10 pictures/slide, to count alveolar macrophages.

\section{In vitro Macrophage Function}

Raw 264.7 cells were obtained from the ATCC (Manassas, VA). The cells were preincubated for $48 \mathrm{hr}$ at $37^{\circ} \mathrm{C}$ and $5 \% \mathrm{CO}_{2}$ under: a. Normal glucose (NG), in DMEM; b. Diabetic milieu (DM) as previously described (29); c. DM + AII (100 nM); d. $\mathrm{DM}+\mathrm{AII}$ (Bachem) (100 nM) and A(1-7) (100 nM); e. DM + AII (100 nM) and AVE 0991 (MedChem Express, Princeton, NJ) (10 nM); f. DM + AII (100 nM) and AVE 0991 (100 nM); g. DM + AII (100 nM) and AVE 0991 (1000 nM). Next, the cells were harvested, incubated for $1 \mathrm{~h}$ with pHrodo and fixed with $4 \%$ formaldehyde solution. Mean fluorescence intensity was determined by flow cytometry using a $\mathrm{BD}^{\mathrm{TM}}$ LSR II flow cytometer.

\section{Statistical Analysis}

GraphPad Prism version 6.0c for Mac OS X (GraphPad Software, San Diego, CA, USA) was used to analyze the data. One-way ANOVA followed by Dunnett's multiple comparisons test were used to compare data. The level of statistical significance was set at $5 \%$. Data are expressed as mean value \pm standard error of the mean (SEM).

\section{RESULTS}

\section{The Overall Diabetic Phenotype of $d b / d b$ Mice Is Not Altered by A(1-7) or NorLeu Treatment for 8 Weeks}

BKS.Cg-Dock $7^{\mathrm{m}}+/+$ Lepr $^{\mathrm{db}} / \mathrm{J}(d b / d b)$ mice develop very severe diabetic phenotype due to overnutrition, closely resembling human disease, which make it a useful tool for studying potential therapeutics $(30,31)$. Mouse weights taken at necropsy show that there is no significant weight reduction after treatment in $d b / d b$ mice (Supplemental Figure 1A). Plasma collected at necropsy after 8 weeks of treatment was used to measure circulating cytokines (TNF- $\alpha$, IL-6, and IL-10) using a multiplex immuneassay. While changes have been noted in these markers with long term (16 weeks) A(1-7) treatment in this model, no significant changes were detected in any of these groups with 8 weeks of treatment (Supplemental Figure 1B) (29).

\section{Diabetic Animals Show Reduced Lung Size, Air Volume Capacity, and Air Volume at Inhalation/Exhalation Ratios, Despite Treatment}

Micro-CT scans of mouse lungs can give insights to the global environment of the lung. In 16-week-old control $d b / d b$ and $h t z$ mice, images of the lung were taken at inhalation and exhalation (Figure 1A). Air volume in the lungs at each measurement was quantified. Consistently, 16-week-old $d b / d b$ animals had lower $\%$ air volume during inhalation and exhalation (Figures 1B,C), even when corrected for total body weight of the mouse. The inhaled/exhaled air volume ratio can be used as a marker for lung elasticity and is also significantly lower in the diabetic animals treated with saline (Figure 1D). A(1-7) treatment-started at 8 weeks of age and continued for 8 weeks-had no effect on this ratio. However, mice treated with NorLeu had a significant improvement in inhalation/exhalation ratios. Although both A(1-7) and NorLeu are thought to target the same receptor, NorLeu has previously shown to have pronounced anti-fibrotic effects beyond those observed after A(1-7) treatments (32).

\section{Diabetic Mice Show Diminished Pathogen Clearance in ex-vivo Neutralization Assays of $S$. aureus While $A(1-7)$ and NorLeu-Treated Diabetic Mice Did Not}

Bacteriocidal activity and PMN function were measured ex-vivo using whole blood assayed for $S$. aureus neutralizing activity. $S$. aureus CFUs were measured before inoculation and after a 30 min incubation with whole blood to calculate the percentage of surviving bacteria (Figure 2A). Blood from htz or A(1-7)treated or NorLeu-treated diabetic animals had significantly lower bacteria levels than that from diabetic saline-treated mice. This suggests the reduced innate immune responses seen in diabetes is ameliorated by Mas agonist treatment.

\section{PMN Function Is Impaired in $d b / d b$ Mouse Model of T2DM and Rescued With A(1-7) or NorLeu Treatment}

To assess PMN function directly, whole blood from $h t z$ and $d b / d b$ mice treated with saline, A(1-7) or NorLeu was incubated with $S$. aureus labeled with a fluorescent tag activated by the low $\mathrm{pH}$ in phagosomes (pHrodo) and read on fluorescent plate reader to determine the kinetic profile of phagocytosis (Figure 2B). Samples from the $h t z$ mice had the highest readout of fluorescence throughout the $110 \mathrm{~min}$ incubation, followed by the $d b / d b$ mice treated with $\mathrm{A}(1-7)$ or NorLeu. Salinetreated $d b / d b$ mice had the lowest emission curve; however, none of the changes in this assay were significant. In the next study, blood samples were collected, WBC were enumerated and incubated with $S$. aureus-pHrodo for $90 \mathrm{~min}$, fixed and analyzed by flow cytometry. PMNs were gated by forward and side scatter and the \% PMNs in total WBCs was measured (Supplemental Figures 1C,D). A calculation was then done to determine the number of total and phagocytic PMNs per $\mathrm{mL}$ of blood (Figure 2C). PMNs in the blood of saline-treated $d b / d b$ mice were less phagocytic compared to the $h t z$ and $\mathrm{A}(1-7)$ - or NorLeu-treated diabetic animals.

\section{Immune Cell Activation Is Affected by T2D and Mas Activation}

Cellular generation of reactive oxygen species (ROS) was measured in the whole blood in the absence and presence of $S$. aureus-pHrodo using CellROX dye (Figure 2D). In the absence of stimulation, blood from all three groups had a similar and constant baseline of CellROX reactivity with the $d b / d b \mathrm{~A}(1-7)$ or NorLeu-treated mice showing the lowest emission level; no significant difference between the study groups was observed. With S. aureus-pHrodo stimulation, blood from all groups had an increase in CellROX reactivity over their unstimulated baselines 


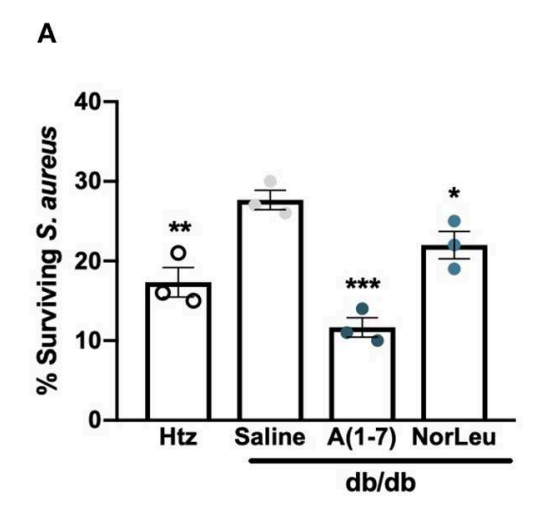

D

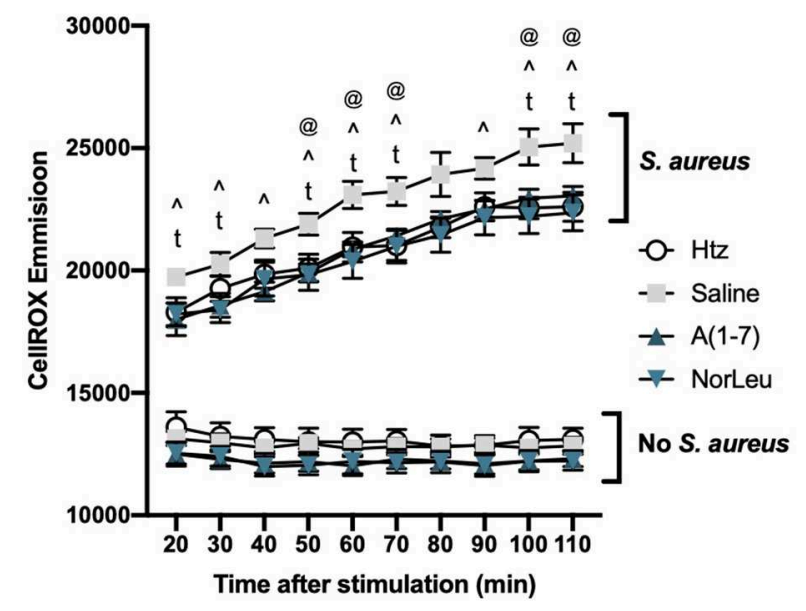

B

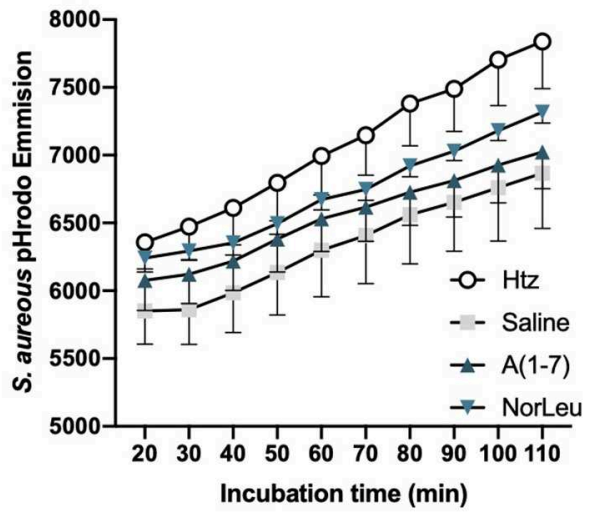

C

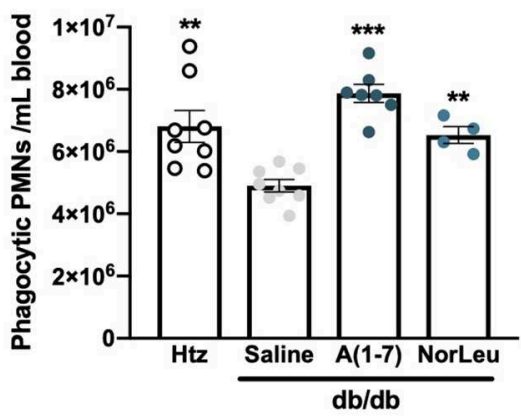

E

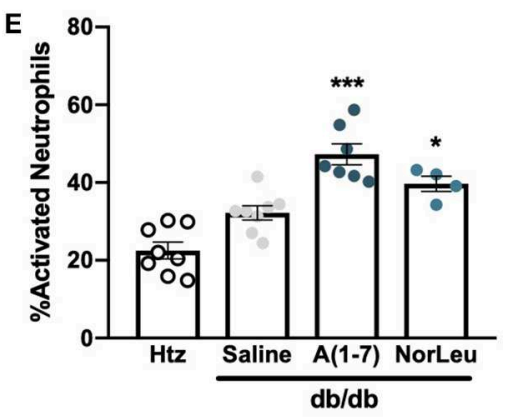

$\mathbf{F}$

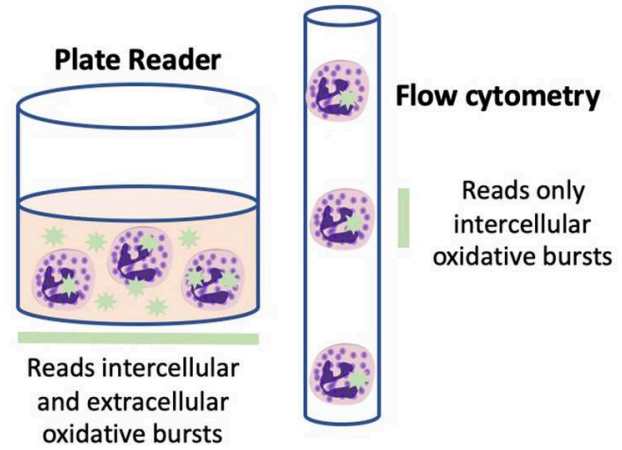

FIGURE 2 | PMNs from $d b / d b$ mice favor degranulation in response to bacterial challenge and those from $A(1-7)$ treated mice favor phagocytosis. Tail-vein blood was collected from htz and $\mathrm{db} / \mathrm{db}$ mice treated with saline or $\mathrm{A}(1-7)$ at $0.5 \mathrm{mg} / \mathrm{kg} /$ day. Whole blood was incubated with live $S$. aureus for $30 \mathrm{~min}$ and the percentage of surviving S. aureus-CFUs was calculated (A). Whole blood samples were also incubated with $S$. aureus-pHrodo; kinetics of phagocytosis were measured using a plate reader with no significant changes between groups at any time period (B), and number of phagocytic PMNs after 90 min (C) was measured using flow cytometry. Blood samples were also incubated with CellROX in the absence and presence of $S$. aureus, the emission was measured with a plate reader for 90 min starting at $20 \mathrm{~min}$ at $10 \mathrm{~min}$ intervals (D) There were only significant differences between treatment groups with $\mathrm{S}$. aureus where saline treated db/db mice were: significantly higher than $h t z$ at $50,60,70,100$, and 110 min ( $\left.{ }^{\circledR}\right)$; significantly higher than $\mathrm{A}(1-7)$ at $20,30,40,50,60,70,90,100$, and 110 min $(\wedge)$; and significantly higher than NorLeu at 20, 30, 50, 60, 70, 90, 100, and $110 \mathrm{~min}\left({ }^{(}\right)$. Further analysis was done on samples incubated with CellROX and S. aureus for 90 min and quantified by flow cytometry (E). Schematic represents the different types of readouts from a plate reader compared to a flow cytometer in regards to CellROX assays (F). Statistics was done using Prism 6 software ANOVA comparing all groups to saline treated $d b / d b ;{ }^{*} p \leq 0.05,{ }^{* *} p \leq 0.01,{ }^{* * *} p \leq 0.001$.

and a constant increase in fluorescence throughout the $110 \mathrm{~min}$ incubation indicating persistent ROS generation. The blood from saline-treated $d b / d b$ mice had the greatest increase in CellROX reactivity over baseline: significantly higher than htz at 50,60, 70,100 , and $110 \mathrm{~min}$; significantly higher than $\mathrm{A}(1-7)$ at 20, 30, $40,50,60,70,90,100$, and $110 \mathrm{~min}$; and significantly higher than NorLeu at 20, 30, 50, 60, 70, 90, 100, and $110 \mathrm{~min}$ (Figure 2D). For flow cytometric analysis, blood samples were stimulated with S. aureus-pHrodo in the presence of CellROX for $90 \mathrm{~min}$, washed, fixed and analyzed by flow cytometric analysis. PMNs were gated as distinct populations by forward and side scatter. Cellular activation in PMNs was measured by CellROX; activation was similar in both htz and saline-treated $d b / d b$ mice. However, significantly higher activation was seen in A(1-7) and NorLeutreated $d b / d b$ mice (Figure 2E).

The differences seen between the plate reader and flow cytometry measurements of CellROX point to functional PMN changes. Samples from saline-treated $d b / d b$ mice analyzed with 

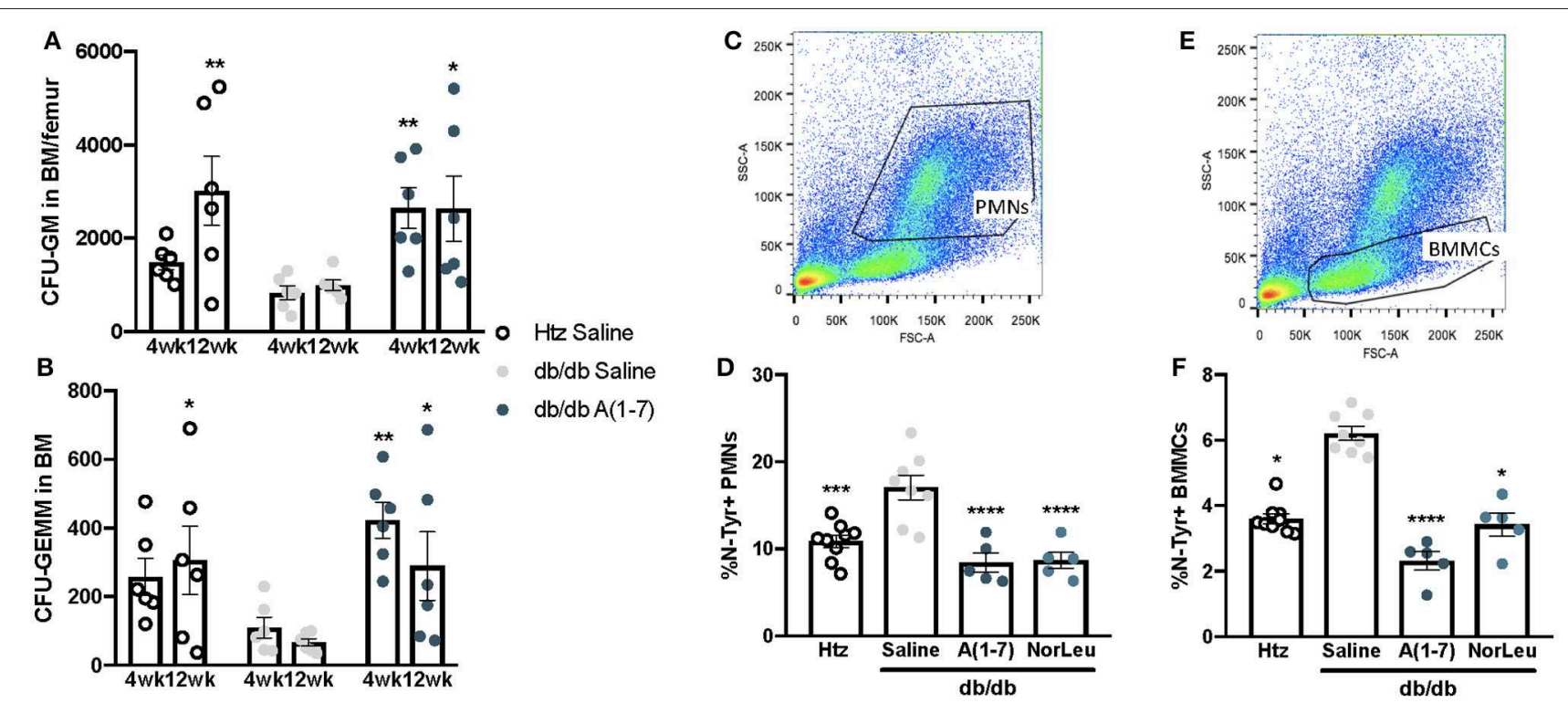

FIGURE 3 | Neutrophil and monocyte progenitors are deficient in $d b / d b$ mice and rescued by $\mathrm{A}(1-7)$ treatment. Neutrophils and monocytes have common progenitor lineage. BM was collected from $h t z$ and $d b / d b$ mice treated with saline and A(1-7) at $0.5 \mathrm{mg} / \mathrm{kg} /$ day for 4 , 8 , and 12 weeks. Cells were cultivated in MethoCult ${ }^{\mathrm{TM}}$ media and both CFU-GEMMs (A) and CFU-GMs (B) were counted per well and multiplied by total number of WBCs in the BM of both femurs. BM cells were also stained with anti-N-Tyr antibody conjugated to FITC and quantified by flow cytometry. This set of animals included $h t z$ controls and $d b / d b$ mice treated with saline or $\mathrm{A}(1-7)$ at $2 \mathrm{mg} / \mathrm{kg} /$ day. Cellular damage by OS was quantified in PMNs (C,D) and BMMCs (E,F) and expressed as percentage of cells positive for intracellular N-Tyr staining. Statistics was done using Prism 6 software ANOVA and compared to saline treated $d b / d b$ mice; ${ }^{*} p \leq 0.05,{ }^{* \star} p \leq 0.01,{ }^{* \star *} p \leq 0.001$, ${ }^{\star \star \star *} p \leq 0.0001$.

the plate reader show the highest CellROX signal, but when analyzed by flow cytometry they have the lowest amount of CellROX signal. This suggests an increase in the production of extracellular ROS through degranulation of PMNs instead of internal pathogen degradation in saline-treated $d b / d b$ mice (Figure 2F). Increases in the degranulation response of PMNs to bacterial challenge may partly explain the higher overall amount of tissue damage frequently seen in infected tissues of diabetic patients. Proper pathogen removal by phagocytosis as opposed to degranulation will reduce the time to clear the infections and reduce the amount of tissue damage (33).

\section{Overall PMN Health May Be Affected Early in Progenitor Development}

PMNs have a high turnover in the blood and are continuously sourced from the BM. Monocytes and neutrophils share common progenitor cells known as CFU-GM and its earlier progenitor CFU-GEMM. As the pathology of diabetes is believed to result from cumulative damage, we measured the CFU-GM and CFUGEMM number in the BM was measured over time. Salinetreated diabetic animals consistently had lower numbers of both CFU-GEMM and CFU-GM, reaching significant difference from both $h t z$ and $d b / d b \mathrm{~A}(1-7)$-treated animals at 12 weeks of treatment (Figures 3A,B). OS was measured in the BM by FITC-anti-N-Tyr staining and quantified by flow cytometry. BM mononuclear cells (BMMCs) (Figures 3C,D) and PMNs (Figures 3E,F) were gated out by forward and side scatter. BMMCs have a lower SSC profile and mostly contain stem cells $\left(\mathrm{CD} 45^{-} \mathrm{Sca}-1^{+}\right)$and other progenitor cells that have not yet started producing CD45 (Supplemental Figure 2); PMNs are appear higher than BMMCs in SSC and consist mostly Neutrophils $\left(\mathrm{CD} 45+\mathrm{Ly}_{6 \mathrm{G}}{ }^{+} \mathrm{Ly}_{6 \mathrm{C}} \mathrm{C}^{\mathrm{lo}}\right)$ and some Eosinophils $\left(\mathrm{CD}_{4}{ }^{+} \mathrm{F} 4 / 80^{+}\right.$Siglec-F $\left.{ }^{+}\right)$. In both BMMCs and PMNs, the saline-treated diabetic animals had a significantly higher percentage of $\mathrm{N}$-Tyr ${ }^{+}$cells; $\mathrm{A}(1-7)$ or NorLeu-treated diabetic animals has levels similar to the $h t z$ group. This suggests an early effect, at the progenitor level, of T2D on PMN and monocyte health; possibly induced by increased OS. A(1-7) and NorLeu treatment may have therapeutic effects very early in innate immune cell development.

\section{Immunity Through Macrophages Is Also Impaired by Diabetic Conditions and Rescued by Mas Activation}

The two most common populations of macrophages in the respiratory tract are AMs and interstitial macrophages (IMs). Although there are molecular markers that are different between these 2 subtypes of pulmonary macrophages, their localization is the most consistent defining characteristic (34-36). In this study AMs, characterized by alveolar localization and morphology, were quantified in sections of lung tissue of non-infected $h t z$ and diabetic mice treated with saline, A(1-7) or NorLeu (Figure 4A). Saline-treated diabetic mice had significantly lower numbers of AMs than $h t z$ mice and $\mathrm{A}(1-7)$ or NorLeu-treated $d b / d b$ mice, suggesting Mas activation protects AMs (Figure 4B). Because of their location, AMs are the first line of defense against many pathogens in the respiratory tract and, if depleted, can account for the delayed pathogen clearance and increase in 

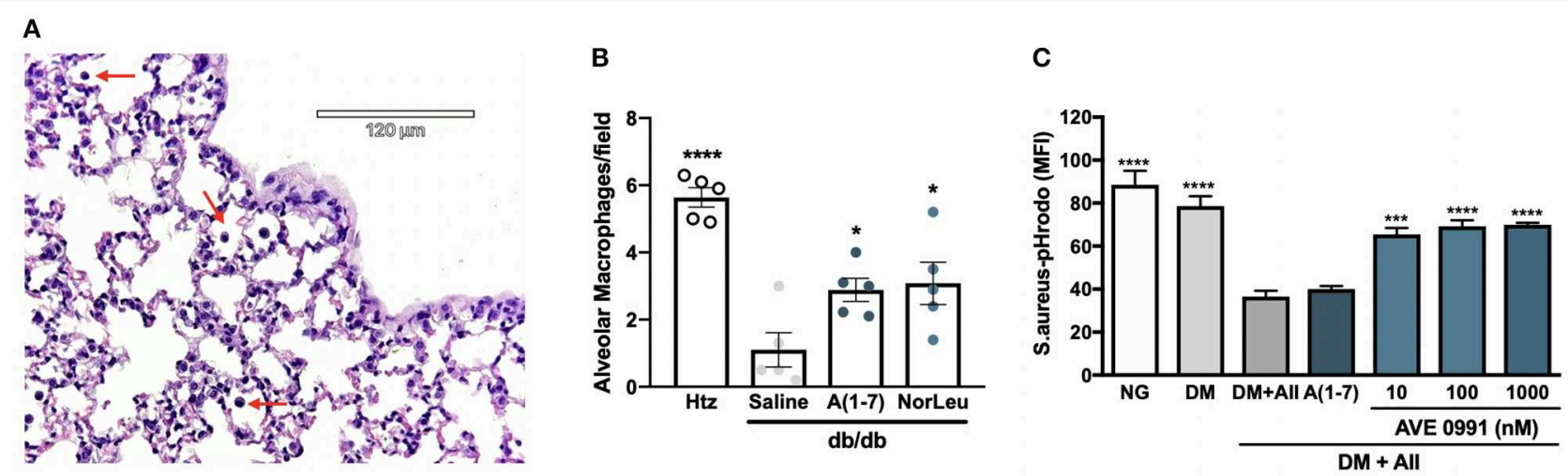

FIGURE 4 | Diabetes altered macrophages respond to Mas-agonists in vivo and in vitro. Pictures were taken of H\&E stained lung sections of $h t z$ and diabetic infection-free animals treated with saline or $\mathrm{A}(1-7)$ at $0.5 \mathrm{mg} / \mathrm{kg} /$ day. A representative picture from a $h$ tz mouse is shown with arrows pointing the AMs (A). AMs were counted per image and an average per frame was calculated for each group (B). In vitro assays were done to quantify the effect or DM and RAS-modification on the phagocytic capabilities of RAW-1 cells (C). Cells were incubated in normal media (NG), media supplemented with 20 mM glucose, endothelin-1 and cortisol (DM), DM media with All every $24 \mathrm{~h}(\mathrm{DM}+\mathrm{A}-\mathrm{II})$, and DM+A-II supplemented every $24 \mathrm{~h}$ with a Mas agonist. After $48 \mathrm{~h}$ in treatment media cells were harvested and incubated with S. aureus-pHrodo, results are shown as MFI for $N=5$ per group; all groups were compared to (DM+All). Statistics was done using Prism 6 software ANOVA; ${ }^{*} p \leq 0.05,{ }^{* * *} p \leq 0.001,{ }^{* * * *} p \leq 0.0001$.

pulmonary infections that are seen in diabetic patients and $d b / d b$ infection model.

\section{Diabetic Conditions Induced in vitro Affect Macrophage Function and Protected by Mas Activation}

RAW-1 cells have long been used to test for in vitro efficacy of novel therapeutics because of their superior consistency in phagocytic assays (37). Here, RAW-1 cells were treated with various media conditions for $48 \mathrm{~h}$ and then incubated with S. aureus-pHrodo to determine their ability to phagocytose pathogens (Figure 4C). Cell incubated with DM show little decrease in phagocytic capabilities unless AII is included. A(17) treatment did not rescue this phenotype, possibly because of its short half-life in media supplemented with FBS. AVE 0991, a small molecule Mas agonist, treatment of RAW-1 cells incubated in DM+AII restored the ability of the RAW1 cells to phagocytose $S$. aureus. Mas activation protected against suppression of phagocytosis in a cell involved in innate immunity in cells exposed to this physiologically relevant diabetic culture medium, further defining Mas as a therapeutic target for diabetic immunosuppression.

\section{DISCUSSION}

Pulmonary infections are a common and significant threat to patients with diabetes. A combination of an impaired immune system and tissue damage increases the chance of a severe complication $(38,39)$. Diminished innate immune function can cause uncontrolled bacterial growth and lead to pneumonia or death (40). Current therapies for T2DM focus on lowering blood glucose, insulin sensitization and weight loss. These treatments are widely used by diabetic patients and, in many cases, effectively lower HbA1c levels; however, many of the diabetic co-morbidities persist despite blood glucose normalization perhaps due to continued increased OS leading to chronic inflammation $(4,6)$. Beyond glycemic control, treatments for diabetic patients need to also focus on the underlying cause of all complications to mitigate the consequences.

Neutrophil degranulation and LPS-induced immune activation have been shown to be negatively affected by hyperglycemia. Leukocytes increase production of ROS in diabetic patients, possibly due to the hyperglycemic environment $(41,42)$. Although hyperglycemia, ROS and inflammation can all affect cell function, existing compounds that decrease ROS levels and inflammation can benefit the patient $(4-7,43)$. Part of the underlying cellular pathological in T2DM is associated with the chronic up-regulation of the pathological arm of the RAS. AII is the main active peptide in this pathological arm and, through binding to AT1, cellular functions are altered leading to an increase in ROS and inflammation. In diabetic patients, AII is known to be upregulated potentially leading to the tissue damage seen in diabetes-related complications (9). Our studies here show that diabetic milieu, including high glucose, cortisol and endothelin 1, is not enough to reduce macrophage phagocytosis in vitro, AII is required; further supporting its key role in the diabetic environment that hinders proper immune function. To decrease the burden of these co-morbidities on both the patient and the health care system, the root cause of the immune dysfunctions and therapies needs to be identified and addressed.

Previous studies as well as those presented here suggest that diabetes impacts innate immunity by hindering the activity of PMNs. Changes in phagocytic capabilities of the PMNs correlated with changes in the ability of PMNs to kill bacteria. Macrophage health is also affected by diabetic conditions. Progenitors to both populations are not only lower in number in diabetic animals, they also exhibit increased OS. All of these pathological changes as a result of diabetes were not observed in $\mathrm{A}(1-7)$ or NorLeu-treated $d b / d b$ mice despite the absence of glucose control. Evidence suggests that this is partly due to reduced OS, a parameter not currently targeted by diabetic 
medications. Treatment with A(1-7) or NorLeu reduced OS and can counteract the pathological effects of AII in various other disease models and is known to have distinct actions according to tissue and pathology $(14,15,43,44)$.

We also noted a shift in cellular response to pathogens in saline-treated diabetic animals compared to the $h t z$ animals. After inoculation with $S$. aureus, in the samples from salinetreated $d b / d b$ mice, ROS was increased in the extracellular environment. Conversely, in $h t z$ and A(1-7) or NorLeu-treated $d b / d b$ mice, these mediators were increased in the intracellular compartments. Since the phagocytic function of PMNs is compromised in diabetes, it may be that the cells are trying to clear pathogens by producing a ROS-rich environment at the site of infection. A(1-7) and NorLeu-treated animals did not share this phenotype, they primarily act through phagocytosis to neutralize bacteria, despite their diabetic status. Focus on extracellular ROS production may help explain the higher amounts of tissue damage seen in T2DM patients since ROS will damage surrounding tissues along with invading pathogens. This neutrophil dysfunction may also be the reason for increased severity of infections $(45,46)$ and delayed wound healing seen in diabetic patients $(47,48)$. Our results are also consistent with other findings that suggest that there is BM dysfunction in diabetic patients (49). Although, $\mathrm{A}(1-7)$ has been shown to decrease inflammation in previous studies (50), our observations were seen in the absence of changes in circulating cytokines, suggesting a direct impact on PMN activity directly impacting tissue health. The exact molecular mechanism by which A(17) acts seems to be dependent on the cell type and insult to the system (50).However, previous studies suggest that the anti-inflammatory actions of RAS-modification are likely mediated by inhibition of NF-kB $(51,52)$ or MAPK (53) signaling pathways.

Taken together, the data presented indicate that RASmodification is a viable therapeutic target for the treatment of diabetic complications, specifically those affected by inadequate innate immune function. Further, these studies show that despite the short half-life of $\mathrm{A}(1-7)$ and NorLeu we can measure changes in innate immunity with once daily dosing. A recent pharmacoeconomic analysis of the effect of inhibitors of the pathological of RAS on pulmonary infections supporting the translatability of these results to diabetic patients. Using a deidentified insurance claims data set it was shown that even newly diagnosed T2DM is associated with higher incidence of pulmonary infections and that RAS-modifying drugs can reduce these outcomes (54). Future studies will explore mechanisms

\section{REFERENCES}

1. Castillo JJ, Castillo JJ, Mull N, Mull N, Reagan JL, Reagan JL, et al. Increased incidence of non-Hodgkin lymphoma, leukemia, and myeloma in patients with diabetes mellitus type 2: a meta-analysis of observational studies. Blood. (2012) 119:4845-50. doi: 10.1182/blood-2011-06-362830

2. Association AD. Economic costs of diabetes in the US in 2012. Diabetes Care. (2013) 36:1033-46. doi: 10.2337/dc12-2625 by which Mas agonism improves immune function in diabetic patients and the role of other immune cells in this paradigm. Ideally, an orally available Mas agonist that replicates the actions of $\mathrm{A}(1-7)$ can be developed as a therapeutic to help improve the patient health in T2DM.

\section{DATA AVAILABILITY STATEMENT}

The datasets generated for this study are available on request to the corresponding author.

\section{ETHICS STATEMENT}

The animal study was reviewed and approved by the University of Southern California Institutional Animal Care and Use Committee.

\section{AUTHOR CONTRIBUTIONS}

All of the authors contributed significantly to the work presented here. MS and KR contributed to the experiment planning, and interpretation of data. MS and KG contributed to the execution of experiments. MS wrote the manuscript. KR and $\mathrm{KG}$ edited the manuscript.

\section{FUNDING}

Research reported in this publication was supported in part by the National Institute of Diabetes and Digestive and Kidney Diseases of the National Institutes of Health under Award Number F31DK103520 and National Institutes of Heart, Lung and Blood under Award Number R01HL082722.

\section{ACKNOWLEDGMENTS}

This work was done with help from members of the Rodgers Lab: Maria I. Banos-Medina, Theresa Espinosa, Alick Tan, Anna Papinska, and Michael Weinberg. We would like to thank them for their help with idea development through discussion and technical aspects of this project.

\section{SUPPLEMENTARY MATERIAL}

The Supplementary Material for this article can be found online at: https://www.frontiersin.org/articles/10.3389/fimmu. 2019.02885/full\#supplementary-material 
6. Rochette L, Zeller M, Cottin Y, Vergely C. Diabetes, oxidative stress and therapeutic strategies. Biochimica et Biophysica Acta. (2014) 1840:2709-29. doi: 10.1016/j.bbagen.2014.05.017

7. Minejima E, Lou M, Nieberg $\mathrm{P}$, Wong-Beringer A. Patients presenting to the hospital with MRSA pneumonia: differentiating characteristics and outcomes with empiric treatment. BMC Infect Dis. (2014) 14:616. doi: 10.1186/1471-2334-14-252

8. Tegegne BS, Habtewold TD, Mengesha MM, Burgerhof JGM. Association between diabetes mellitus and multi-drug-resistant tuberculosis: a protocol for a systematic review and meta-analysis. Syst Rev. (2017) 6:44. doi: 10.1186/s13643-017-0407-9

9. Yang J, Tan Y, Zhao F, Ma Z, Wang Y, Zheng S, et al. Angiotensin II plays a critical role in diabetic pulmonary fibrosis most likely via activation of NADPH oxidase-mediated nitrosative damage. Am J Physiol Endocrinol Metab. (2011) 301:E132-44. doi: 10.1152/ajpendo.00629.2010

10. Ogihara T, Asano T, Ando K, Chiba Y, Sakoda H, Anai M, et al. Angiotensin II-induced insulin resistance is associated with enhanced insulin signaling. Hypertension. (2002) 40:872-9. doi: 10.1161/01.HYP.0000040262.48405.A8

11. Wei Y, Sowers JR, Clark SE, Li W. Angiotensin II-induced skeletal muscle insulin resistance mediated by NF- $\kappa$ B activation via NADPH oxidase. Am J Physiol Endocrinol Metab. (2008) 294:E345-51. doi: 10.1152/ajpendo.00456.2007

12. Ferder L, Inserra F, Martínez-Maldonado M. Inflammation and the metabolic syndrome: role of angiotensin II and oxidative stress. Curr Hypertens Rep. (2006) 8:191-8. doi: 10.1007/s11906-006-0050-7

13. Liu C, Lv X-H, Li H-X, Cao X, Zhang F, Wang L, et al. Angiotensin- (1-7) suppresses oxidative stress and improves glucose uptake via Mas receptor in adipocytes. Acta Diabetol. (2011) 49:291-9. doi: 10.1007/s00592-011-0348-z

14. Machiela MJ, Lindstrom S, Allen NE, Haiman CA, Albanes D, Barricarte A, et al. Association of type 2 diabetes susceptibility variants with advanced prostate cancer risk in the breast and prostate cancer cohort consortium. Am J Epidemiol. (2012) 176:1121-9. doi: 10.1093/aje/kws191

15. Wie S-H, So BH, Song JY, Cheong HJ, Seo YB, Choi SH, et al. A comparison of the clinical and epidemiological characteristics of adult patients with laboratory-confirmed influenza A or B during the 2011-2012 Influenza Season in Korea: a multi-center study. PLoS ONE. (2013) 8:e62685. doi: 10.1371/journal.pone.0062685

16. Huang J, Xiao Y, Zheng P, Zhou W, Wang Y, Huang G, et al. Distinct neutrophil counts and functions in newly diagnosed type 1 diabetes, latent autoimmune diabetes in adults, and type 2 diabetes. Diabetes Metab Res Rev. (2019) 35:e3064. doi: 10.1002/dmrr.3064

17. Bryk AH, Prior SM, Plens K, Konieczynska M, Hohendorff J, Malecki MT, et al. Predictors of neutrophil extracellular traps markers in type 2 diabetes mellitus: associations with a prothrombotic state and hypofibrinolysis. Cardiovasc Diabetol. (2019) 18:1-12. doi: 10.1186/s12933-019-0850-0

18. Kapoula GV, Kontou PI, Bagos PG. Diagnostic accuracy of neutrophil gelatinase-associated lipocalin for predicting early diabetic nephropathy in patients with type 1 and type 2 diabetes mellitus: a systematic review and meta-analysis. J Appl Lab Med. (2019) 4:78-94. doi: 10.1373/jalm.2018.028530

19. Angkananard $T$, Anothaisintawee $T$, Ingsathit A, McEvoy M, Silapat $\mathrm{K}$, Attia J, et al. Mediation effect of neutrophil lymphocyte ratio on cardiometabolic risk factors and cardiovascular events. Sci Rep. (2019) 9:261811. doi: 10.1038/s41598-019-39004-9

20. Giorgino F, Leonardini A, Laviola L. Cardiovascular disease and glycemic control in type 2 diabetes: now that the dust is settling from large clinical trials. Ann NY Acad Sci. (2013) 1281:36-50. doi: 10.1111/nyas.12044

21. Rodgers KE, Bolton LL, Verco S, Dizerega GS. NorLeu3-Angiotensin (1-7) [DSC127] as a therapy for the healing of diabetic foot ulcers. Adv Wound Care. (2015) 4:339-45. doi: 10.1089/wound.2014.0609

22. Rodgers KE, Roda N, Felix JE, Espinoza T, Maldonado S, diZerega G. Histological evaluation of the effects of angiotensin peptides on wound repair in diabetic mice. Exp Dermatol. (2003) 12:784-90. doi: 10.1111/j.0906-6705.2003.00087.x

23. Rodgers K, Verco S, Bolton L, diZerega G. Accelerated healing of diabetic wounds by NorLeu (3)-angiotensin (1-7). Exp Opin Investig Drugs. (2011) 20:1575-81. doi: 10.1517/13543784.2011.619976

24. Balingit PP, Armstrong DG, Reyzelman AM, Bolton L, Verco SJ, Rodgers KE, et al. NorLeu3-A(1-7) stimulation of diabetic foot ulcer healing: results of a randomized, parallel-group, double-blind, placebocontrolled phase 2 clinical trial. Wound Repair Regen. (2012) 20:482-90. doi: 10.1111/j.1524-475X.2012.00804.X

25. Jadhav SS, Sharma N, Meeks CJ, Mordwinkin NM, Espinoza TB, Roda NR, et al. Effects of combined radiation and burn injury on the renin-angiotensin system. Wound Repair Regen. (2013) 21:131-40. doi: 10.1111/j.1524-475X.2012.00867.x

26. Martin TR, Frevert CW. Innate Immunity in the Lungs. Proc Am Thorac Soc. (2012) 2:403-11. doi: 10.1513/pats.200508-090JS

27. Craig A, Mai J, Cai S, Jeyaseelan S. Neutrophil recruitment to the lungs during bacterial pneumonia. Infect Immun. (2009) 77:568-75. doi: 10.1128/IAI.00832-08

28. Mori J, Patel VB, Ramprasath T, Alrob OA, DesAulniers J, Scholey JW, et al. Angiotensin 1-7 mediates renoprotection against diabetic nephropathy by reducing oxidative stress, inflammation, and lipotoxicity. Am J Physiol Renal Physiol. (2014) 306:F812-21. doi: 10.1152/ajprenal.00655.2013

29. Papinska AM, Soto M, Meeks CJ, Rodgers KE. Long-term administration of angiotensin (1-7) prevents heart and lung dysfunction in a mouse model of type 2 diabetes $(\mathrm{db} / \mathrm{db})$ by reducing oxidative stress, inflammation and pathological remodeling. Pharmacol Res. (2016) 107:372-80. doi: 10.1016/j.phrs.2016.02.026

30. Vedova Della MC, Muñoz MD, Santillan LD, Plateo-Pignatari MG, Germanó MJ, Rinaldi Tosi ME, et al. A Mouse model of diet-induced obesity resembling most features of human metabolic syndrome. Nutr Metab Insights. (2016) 9:93-102. doi: 10.4137/NMI.S32907

31. Guest PC, Rahmoune H. Characterization of the $\mathrm{db} / \mathrm{db}$ mouse model of type 2 diabetes. Methods Mol Biol. (2019) 1916:195-201. doi: 10.1007/978-1-4939-8994-2_18

32. Rodgers KE, Ellefson DD, Espinoza T, Roda N, Maldonado S, Dizerega GS. Effect of NorLeu3-A(1-7) on scar formation over time after fullthickness incision injury in the rat. Wound Repair Regen. (2005) 13:309-17. doi: 10.1111/j.1067-1927.2005.130314.x

33. Soehnlein O, Steffens S, Hidalgo A, Weber C. Neutrophils as protagonists and targets in chronic inflammation. Nat Rev Immunol. (2017) 17:248-61. doi: 10.1038/nri.2017.10

34. Naeem A, Rai SN, Pierre L. Histology, alveolar macrophages. In: StatPearls. Treasure Island, FL: StatPearls Publishing (2019).

35. Lambrecht BN. Alveolar macrophage in the driver's seat. Immunity. (2006) 24:366-8. doi: 10.1016/j.immuni.2006.03.008

36. Guilliams M, De Kleer I, Henri S, Post S, Vanhoutte L, De Prijck S, et al. Alveolar macrophages develop from fetal monocytes that differentiate into long-lived cells in the first week of life via GM-CSF. J Exp Med. (2013) 210:1977-92. doi: 10.1084/jem.20131199

37. Taciak B, Białasek M, Braniewska A, Sas Z, Sawicka P, Kiraga E, et al. Evaluation of phenotypic and functional stability of RAW 264.7 cell line through serial passages. PLoS ONE. (2018) 13:e0198943. doi: 10.1371/journal.pone.0198943

38. Schmidt AM, Yan SD, Yan SF, Stern DM. The multiligand receptor RAGE as a progression factor amplifying immune and inflammatory responses. J Clin Investig. (2001) 108:949-55. doi: 10.1172/JCI200114002

39. Graves DT, Kayal RA. Diabetic complications and dysregulated innate immunity. Front Biosci. (2008) 13:1257. doi: 10.2741/2757

40. Mooney JP, Galloway LJ, Riley EM. Malaria, anemia, and invasive bacterial disease: a neutrophil problem? J Leukoc Biol. (2019) 105:645-55. doi: 10.1002/JLB.3RI1018-400R

41. Caramori ML, Mauer M. Diabetes and nephropathy. Curr Opin Nephrol Hypertens. (2003) 12:273-82. doi: 10.1097/00041552-20030500000008

42. Arcidiacono B, Iiritano S, Nocera A, Possidente K, Nevolo MT, Ventura V, et al. Insulin resistance and cancer risk: an overview of the pathogenetic mechanisms. Exp Diabetes Res. (2012) 2012:1-12. doi: 10.1155/2012/7 89174

43. Mordwinkin NM, Meeks CJ, Jadhav SS, Espinoza T, Roda N, diZerega GS, et al. Angiotensin- (1-7) administration reduces oxidative stress in diabetic bone marrow. Endocrinology. (2012) 153:2189-97. doi: 10.1210/en.2011-2031

44. Chen Q, Yang Y, Huang Y, Pan C, Liu L, Qiu H. Angiotensin- (1-7) attenuates lung fibrosis by way of Mas receptor in acute lung injury. J Surg Res. (2013) 185:740-7. doi: 10.1016/j.jss.2013.06.052 
45. Shah BR, Hux JE. Quantifying the risk of infectious diseases for people with diabetes. Diabetes Care. (2003) 26:510-3. doi: 10.2337/diacare. 26.2.510

46. Farnsworth CW, Shehatou CT, Maynard R. A humoral immune defect distinguishes the response to Staphylococcus aureus infections in mice with obesity and type 2 diabetes from that in mice with type 1 diabetes. Infect Immun. (2015) 83:2264-74. doi: 10.1128/IAI. 03074-14

47. Wicks K, Torbica T, Mace KA. Myeloid cell dysfunction and the pathogenesis of the diabetic chronic wound. Semin Immunol. (2014) 26:34153. doi: 10.1016/j.smim.2014.04.006

48. Wong SL, Demers M, Martinod K, Gallant M, Wang Y, Goldfine $\mathrm{AB}$, et al. Diabetes primes neutrophils to undergo NETosis, which impairs wound healing. Nat Med. (2015) 21:815-9. doi: 10.1038/ nm. 3887

49. Ito S, Ohno Y, Tanaka T, Kobuchi S, Ayajiki K, Manabe E, et al. Neutrophil/lymphocyte ratio elevation in renal dysfunction is caused by distortion of leukocyte hematopoiesis in bone marrow. Renal Failure. (2019) 41:284-93. doi: 10.1080/0886022X.2019.1597736

50. Passos-Silva DG, Verano-Braga T, Santos RAS. Angiotensin- (17): beyond the cardio-renal actions. Clin Sci. (2012) 124:443-56. doi: 10.1042/CS20120461

51. Fang Y, Gao F, Liu Z. Angiotensin-converting enzyme 2 attenuates inflammatory response and oxidative stress in hyperoxic lung injury by regulating NF-кB and Nrf2 pathways. QJM. (2019) 58:119. doi: 10.1093/qjmed/hcz206
52. Huang W, Cao Y, Liu Y, Ping F, Shang J, Zhang Z, et al. Activating Mas receptor protects human pulmonary microvascular endothelial cells against LPS-induced apoptosis via the NF-kB p65/P53 feedback pathways. J Cell Physiol. (2019) 234:12865-75. doi: 10.1002/jcp.27951

53. Chen Q, Liu J, Wang W, Liu S, Yang X, Chen M, et al. Sini decoction ameliorates sepsis-induced acute lung injury via regulating ACE2-Ang (1-7)-Mas axis and inhibiting the MAPK signaling pathway. Biomed Pharmacotherap. (2019) 115:108971. doi: 10.1016/j.biopha.2019.108971

54. Soto M, Bang SI, McCombs J, Rodgers KE. Renin Angiotensin systemmodifying therapies are associated with improved pulmonary health. Clin Diabetes Endocrinol. (2017) 3:6. doi: 10.1186/s40842-017-0044-1

Disclaimer: The content is solely the responsibility of the authors and does not necessarily represent the official views of the National Institutes of Health.

Conflict of Interest: The authors declare that the research was conducted in the absence of any commercial or financial relationships that could be construed as a potential conflict of interest.

Copyright (c) 2019 Soto, Gaffney and Rodgers. This is an open-access article distributed under the terms of the Creative Commons Attribution License (CC BY). The use, distribution or reproduction in other forums is permitted, provided the original author(s) and the copyright owner(s) are credited and that the original publication in this journal is cited, in accordance with accepted academic practice. No use, distribution or reproduction is permitted which does not comply with these terms. 